\title{
Cratylia mollis 1, 4 Lectin: A New Biotechnological Tool in IL-6, IL-17A, IL-22, and IL-23 Induction and Generation of Immunological Memory
}

\author{
Priscilla Stela Santana de Oliveira, ${ }^{1}$ Moacyr Jesus Barreto de Melo Rêgo, ${ }^{1}$ \\ Rafael Ramos da Silva, ${ }^{1}$ Mariana Brayner Cavalcanti, ${ }^{1}$ Suely Lins Galdino, ${ }^{1}$ \\ Maria Tereza dos Santos Correia, ${ }^{2}$ Luana Cassandra Breitenbach Barroso Coelho, ${ }^{2}$ \\ and Maira Galdino da Rocha Pitta ${ }^{1}$ \\ ${ }^{1}$ Laboratório de Imunomodulação e Novas Abordagens Terapêuticas (LINAT), Universidade Federal de Pernambuco (UFPE), \\ Avenida Prof. Moraes Rêgo 1235, Cidade Universitária, 50670-901 Recife, PE, Brazil \\ ${ }^{2}$ Laboratório de Glicoproteínas, Centro de Ciências Biológicas, Universidade Federal de Pernambuco (UFPE), \\ Avenida Prof. Moraes Rêgo 1235, Cidade Universitária, 50670-901 Recife, PE, Brazil
}

Correspondence should be addressed to Maira Galdino da Rocha Pitta; mgrpitta@gmail.com

Received 21 November 2012; Revised 30 January 2013; Accepted 10 February 2013

Academic Editor: Marija Mostarica-Stojković

Copyright (c) 2013 Priscilla Stela Santana de Oliveira et al. This is an open access article distributed under the Creative Commons Attribution License, which permits unrestricted use, distribution, and reproduction in any medium, provided the original work is properly cited.

Cratylia mollis lectin has already established cytokine induction in Th1 and Th2 pathways. Thereby, this study aimed to evaluate Cramoll 1, 4 in IL-6, IL-17A, IL-22, and IL-23 induction as well as analyze immunologic memory mechanism by reinducing lymphocyte stimulation. Initially we performed a screening in cultured splenocytes where Cramoll 1, 4 stimulated IL-6 production $5 \mathrm{x}$ more than ConA $(P<0.05)$. The same behavior was observed with IL- 22 where the increase was greater than $4 \mathrm{x}$. Nevertheless, IL-17A induction was similar for both lectins. In PBMCs, the same splenocytes course was observed for IL-6 and IL-17A. Concerning the stimulation of IL-22 and IL-23 Cramoll 1, 4 was more efficient than ConA in cytokines stimulation mainly in IL-23 $(P<0.01)$. Analyzing reinduced lymphocyte stimulation, IL-17A production was higher $(P<0.001)$ when the first stimulus was realized with Cramoll 1,4 at $1 \mu \mathrm{g} / \mathrm{mL}$ and the second at $5 \mu \mathrm{g} / \mathrm{mL}$. IL-22 shows significant differences $(P<0.01)$ at the same condition. Nevertheless, IL-23 revels the best response when the first stimuli was realized with Cramoll1, 4 at $100 \mathrm{ng} / \mathrm{mL}$ and the second with $5 \mu \mathrm{g} / \mathrm{mL}$. We conclude that the Cramoll 1,4 is able to induce IL-6, IL-17A, IL-22, and IL-23 cytokines in vitro better than Concavalin A, besides immunologic memory generation, being a potential biotechnological tool in Th17 pathway studies.

\section{Introduction}

Cramoll lectins are purified from Cratylia mollis leguminous seeds; until now four isoforms were discovered on this species, among then the association of forms 1 and 4 (Cramoll 1, 4). Like the well-established lectin from Canavalia ensiformis, Concanavalin A (ConA), Cramoll has its carbohydrate recognition site specific for glucose/mannose. Isoforms 1 and 1, 4 have similar characteristics to ConA [1] not only in its structural arrangement but also on its in vitro activity as $\mathrm{T}$ lymphocyte mitogen [2].

Studies comparing Cramoll 1, 4 and ConA have proved that the high biotechnological potential of Cramoll 1, 4 in relation to ConA such as tumor molecular probes, acting as breast [3], prostate cancer biomarker [4], and in vitro induction of cytokines belonging to the classical Th1 immune pathway in mice splenocytes $[5,6]$. Furthermore Cramoll 1, 4 also showed healing properties in mouse induced lesions 
[7], anticancer effects, when encapsulated into liposomes [8], and induced epimastigotes death by necrosis in an in vitro tripanosomiasis model [9].

On the other hand the exact role of Cramoll and ConA in the induction of cytokines belonging to Th17 pathway has not been reported yet [10]. In this pathway, the synergistic action of IL-6 and transforming growth factor beta (TGF- $\beta$ ) on naive $\mathrm{T}$ cells, induce differentiation into Th17 lymphocytes through the activation of transcription factor regulator (ROR- $\gamma \mathrm{t}$ ) becoming essentially producers of IL17A, IL-17F, and IL-22, with IL-23 being responsible for the phenotype maintaining [11]. The Th17 pathway mediates protective actions in bacterial and fungal infections, but in human autoimmune diseases it has deleterious effects compromising the patient's clinical evolution, becoming a great target on experimental research [12]. Therefore, this study aimed to evaluate the stimulation effectiveness of ConA and Cramoll 1, 4 in IL-6, IL-17A, IL-22, and IL-23 production as well as analyze the mechanism of immunologic memory boosting a secondary response primary immunization by Cramoll 1, 4 .

\section{Materials and Methods}

2.1. Lectins. Seeds of C. mollis Mart. (camaratu bean) were collected in the State of Pernambuco (Brazil), and the lectin (Cramoll 1, 4) was purified according to Correia and Coelho, 1995 [13]. The seed extract $(10 \% \mathrm{w} / \mathrm{v}$ in $0.15 \mathrm{M} \mathrm{NaCl})$ was ammonium sulphate fractionated (40-60\%) and then purified by affinity chromatography on a Sephadex G-75 column. Cramoll 1, 4 elution was performed with $0.3 \mathrm{M}$ glucose in $0.15 \mathrm{M} \mathrm{NaCl}$. ConA was purchased from Sigma-Aldrich.

2.2. Animals. Experimental assays utilized mice BALB/c (male, 45 days old). The animals $(n=6)$ were raised and maintained at Laboratório de Imunopatologia Keizo Asami (LIKA), Universidade Federal de Pernambuco (UFPE), Recife, Brazil. The guidelines of the Animals' Ethical Committee of our institution were followed after their approval.

2.3. Preparation of Splenocytes. The spleen of each mouse was removed aseptically and placed in a Petri dish containing RPMI-1640 (Gibco). In a vertical flow, each spleen was transferred to another Petri dish where they were soaked. The cell suspensions obtained from each spleen were filtered in a $40 \mu \mathrm{m}$ nylon cell strainer (BD bioscience) and then transferred to centrifuge tubes. Spleen homogenates were then centrifuged twice at $300 \mathrm{~g}$ for $10 \mathrm{~min}$. Cells were then treated with 1X RBC lysis buffer (eBiosciences). Cells were counted in a Neubauer chamber, and cell viability was determined by the trypan blue exclusion method being used when viability was $>98 \%$.

2.4. PBMCs Purification. Peripheral blood mononuclear cells (PBMC) were obtained from heparinized blood from healthy, nonsmoking donors who had not taken any drugs for at least 15 days prior to sampling that was collected $(n=$ 6 ), and the PBMC were isolated via a standard method of density-gradient centrifugation over Ficoll-Hypaque (GE Healthcare). Cells were counted in a Neubauer chamber, and cell viability was determined by trypan blue exclusion method. Cells were only used when viability was $>98 \%$. All donors signed an informed consent form, and the study was approved by the human research ethics committee of UFPE Health Sciences Center (CEP/CCS/UFPE N0 145/09).

2.5. Splenocytes and PBMCs Cultures. Splenocytes and PBMC $\left(1 \times 10^{6}\right.$ cell/well) were cultured in 24 -well plates in RPMI-1640 (Gibco) supplemented with $10 \%$ fetal bovine serum (Gibco), HEPES $10 \mathrm{mM}$ (Gibco), and penicillin/ streptomycin $200 \mathrm{U} / \mathrm{mL}$ (Gibco). The cells were stimulated with ConA and Cramoll 1,4 at $100 \mathrm{ng} / \mathrm{mL}, 1 \mu \mathrm{g} / \mathrm{mL}$, and $5 \mu \mathrm{g} / \mathrm{mL}$ or not and incubated at $37^{\circ} \mathrm{C}$ in a humidified $5 \% \mathrm{CO}_{2}$ incubator. Unstimulated (USC) cells were used as control.

2.6. Cytokine Titration. Cytokines in the supernatants of splenocyte cultures were assayed with ELISA mouse kits according to the manufacturer's instructions. The lower limits of ELISA kits detection were $7.8 \mathrm{pg} / \mathrm{mL}$ for IL-6 (BD Biosciences), $3.9 \mathrm{pg} / \mathrm{mL}$ for IL-17A (eBiosciences), $7.8 \mathrm{pg} / \mathrm{mL}$ from IL-22 (eBiosciences), and 15.6 from IFN $\gamma$ (BD Biosciences). Cytokines were assayed in PBMCs culture from healthy subjects with ELISA human kits according to the manufacturer's instructions. The lower limits of detection for the ELISA human kits were $15.6 \mathrm{pg} / \mathrm{mL}$ for IL-6 (BD Biosciences), $3.9 \mathrm{pg} / \mathrm{mL}$ for IL-17A (eBiosciences), $15.6 \mathrm{pg} / \mathrm{mL}$ for IL-22 (eBiosciences), and 15.62 for IL-23p19 (eBiosciences) levels were measured at $48 \mathrm{~h}$ and $24 \mathrm{~h}$ after restimulation by human kits.

2.7. Statistical Analysis. All experiments were performed at least three independent times before statistical analysis. The test used was one-way ANOVA in which differences were considered significant when $P<0.05$. In all graphs, bars represent mean value \pm standard deviation.

\section{Results}

3.1. Cramoll 1, 4 Induces IL-6, IL-17A, and IL-22 Production in $B A L B / c$ Splenocytes Cultures. Initially, a Cramoll 1, 4 cytotoxicity assay was performed, and no toxicity was observed in all tested concentrations $(100 \mathrm{ng} / \mathrm{mL}-5 \mu \mathrm{g} / \mathrm{mL})$. These data were previously confirmed by our group [14]. When the ability of Cramoll 1, 4 to induce IL-6, IL-17A, IL22 cytokine production was evaluated, a significant increased production of IL- 6 and IL-22 at all concentrations tested was obtained when compared with ConA (Figure 1). The best dose to stimulate cytokine production was $5 \mu \mathrm{g} / \mathrm{mL}$ where IL-6 production by Cramoll 1,4 stimulus $(279.8 \mathrm{pg} / \mathrm{mL})$ was 5 times higher than ConA $(53.4 \mathrm{pg} / \mathrm{mL}) P<0.05$ (Figure 1(a)). The same behavior was observed for IL-22 which production was $117.6 \mathrm{pg} / \mathrm{mL}$ when stimulated with Cramoll 1, 4 and $30.5 \mathrm{pg} / \mathrm{mL}$ when stimulated with ConA revealing that Cramoll has 3.8 times more induction power of IL-22 production (Figure 1(b)), although the difference was not statistically significant. On the other hand, similar 


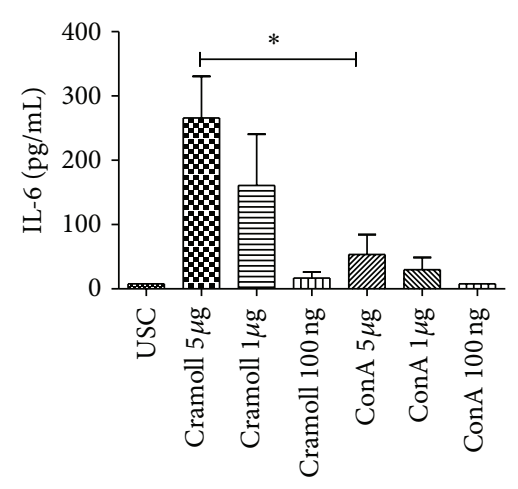

(a)

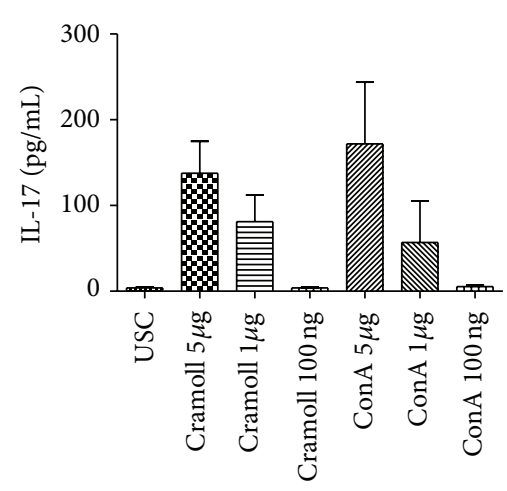

(b)

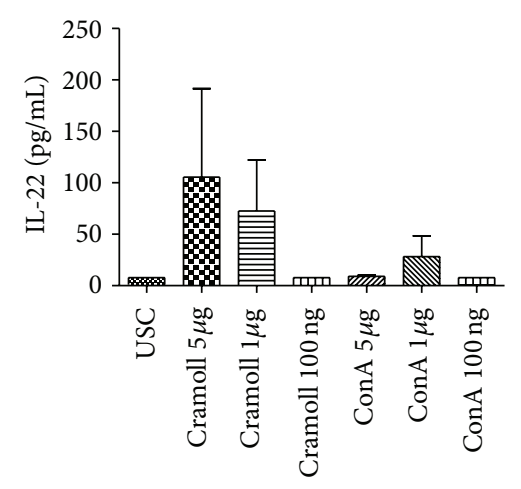

(c)

FIGURE 1: Production of IL-6 (a), IL-17 (b), and IL-22 (c) cytokines (pg/mL) from BALB/c mice splenocyte cultures in relation to different stimuli concentrations of Cramoll 1, 4 and ConA.

results between Cramoll 1, 4 and ConA were found in IL-17A production $(145.4 \mathrm{pg} / \mathrm{mL}$ and $117.2 \mathrm{pg} / \mathrm{mL})$, respectively (Figure $1(\mathrm{c})$ ). No IL-17A significative differences were observed to both lectins in assayed concentration.

3.2. Cramoll 1, 4 Induces IL-6, IL-17A, IL-22, and IL-23 Production in PBMC Culture. The confirmation of Th17 pathway cytokine production in murine splenocytes triggered our interest in analyzing the effects of Cramoll 1, 4 and ConA in human PBMCs. Cramoll 1, 4 was effective in stimulating the production of IL- 6 better than ConA in all tested concentration being significative at $5 \mu \mathrm{g}(P<0.01)$ and $1 \mu \mathrm{g}(P<$ 0.05) (Figure 2(a)). For IL-17A (Figure 2(b)) Cramoll 1, 4 was more effective to stimulate cytokine production in all tested concentration, but no result was statistically significative (Figure 2(b)). The stimulation of IL-22 (Figure 2(c)), that with IL-17A form the main cytokines produced by Th17, was more effective with Cramoll 1, 4 than ConA. Cramoll 1, 4 was also more efficient than ConA in IL-23p19 stimulation. This cytokine is responsible for maintenance of Th17 pathway which production by Cramoll 1,4 on $5 \mu \mathrm{g} / \mathrm{mL}$ was $167 \mathrm{pg} / \mathrm{mL}$ and for ConA was $15 \mathrm{pg} / \mathrm{mL}(P<0.01)$ showing a high significant difference.

3.3. Cramoll 1, 4 Induces Immunological Memory. Assays involving a protocol of previous stimulus followed by a second stimulus can supply information in relation to the immunological memory that the target compound is capable of inducing an in vitro memory. Then, after five days of first incubation with different lectin concentrations, the second stimulation was performed during $24 \mathrm{~h}$ before cytokines dosage. Figure 3(a) reveals that when Cramoll 1, 4 and ConA are used as unique stimuli for 6 days (five days +24 h without second stimulus), Cramoll 1, 4 is more efficient than ConA in stimulating IL-6. The same behavior was obtained when ConA and Cramoll 1, 4 were used as a second stimulus at all concentrations tested; the first and second stimuli with Cramoll 1, 4 were the major IL-6 inductor. IL-17 production showed significant differences in the statistical analysis $(P<$ 0.001) only when the first stimulus was performed with
Cramoll 1,4 at $1 \mu \mathrm{g} / \mathrm{mL}$ and the second with Cramoll 1, 4 at $5 \mu \mathrm{g} / \mathrm{mL}$ (Figure 3(b)). The same behavior was accompanied by IL-22 that only showed significant differences $(P<0.01)$ at the same condition (Figure 3(c)). The analysis of IL-23 data revealed an interesting result; the best response was obtained when the first stimuli was performed with Cramoll 1, 4 at $100 \mathrm{ng} / \mathrm{mL}$ and the second $5 \mu \mathrm{g} / \mathrm{mL}$ (Figure 3(d)) differing from the results obtained by other interleukins analyzed in this study. But like IL-17A and IL-22 there was a significant difference when these IL-23 data were compared with ConA.

\section{Discussion}

Our hypothesis based on IL-6, IL-17A, IL-22, and IL-23 stimulations by lectins is well grounded on the literature that shows many lectins with immunomodulatory effects. The lectin abrin, extracted from Abrus precatorius plant, induces murine splenocyte proliferation leading to a Th1 response, activation of NK cells, and stimulation of peritoneal macrophages [15]. Also, the lectin Abrus agglutinin (AAG) showed Th1 type immunomodulatory response [16]. Even the stimulatory effect of the Th1 pathway by Cramoll 1, 4 was reported in the literature [5]. These lectin effects have been heavily studied since the 1980s and 1990s [17-19]; however, no work so far observed the potential effect of these molecules towards Th17 pathway.

Initially, we assayed the ability of Cramoll 1, 4 to induce IL-6, IL-17A, and IL-22 secretions in mice splenocytes. IL-6 is a proliferative cytokine, stimulatory of immune cells mainly in the $\mathrm{T}$ cells mediated immune response and also, could act as an inductive cytokine of Th17 cell polarization, which in turn produces mainly IL-17 and IL-22 [20, 21]. Cramoll 1, 4 itself has been identified as an immunomodulator in splenocyte cultures from rats treated intraperitoneally with this lectin, increasing ROS production, calcium levels, and expression of IL1B mRNA but not IL-6 or IL-10 [22]. These IL-6 data analyses corroborate with our results. Other work from our group showed that Cramoll 1, 4 at $10 \mu \mathrm{g} / \mathrm{mL}$ revealed higher INF- $\gamma$ production when compared with ConA and revealed an anti-inflammatory response through NO suppression [5]. Recently Abreu and colleagues [23] 


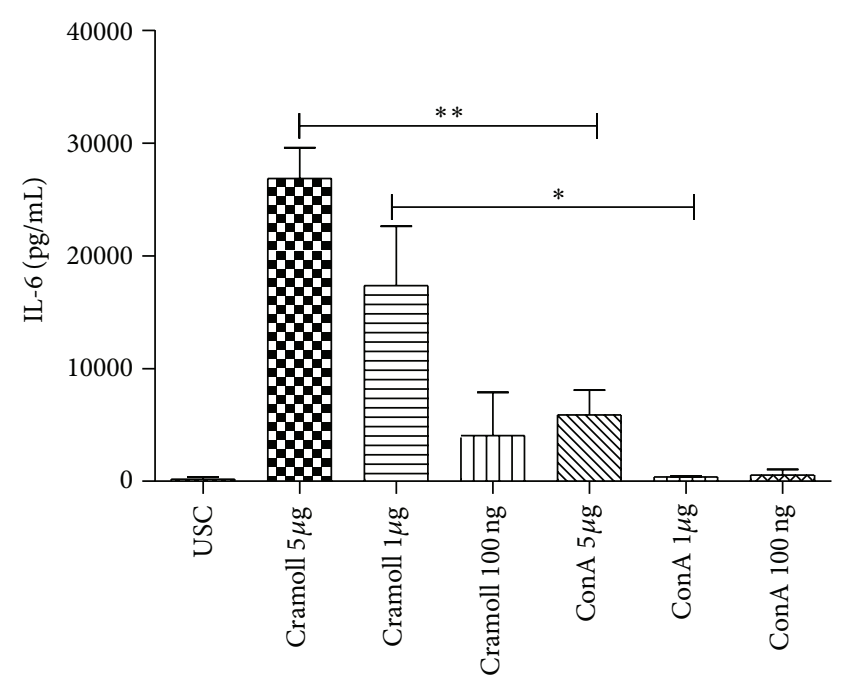

(a)

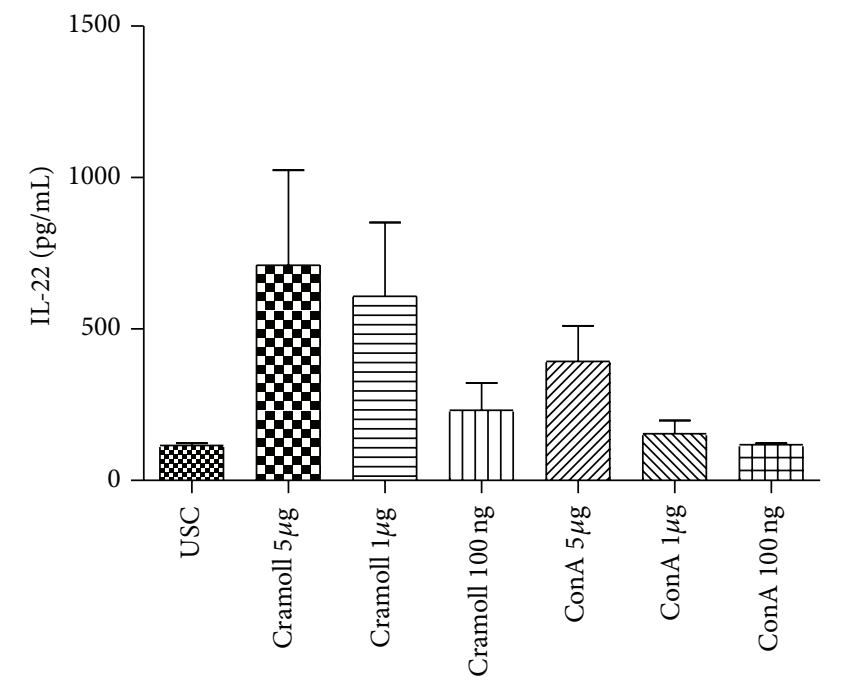

(c)

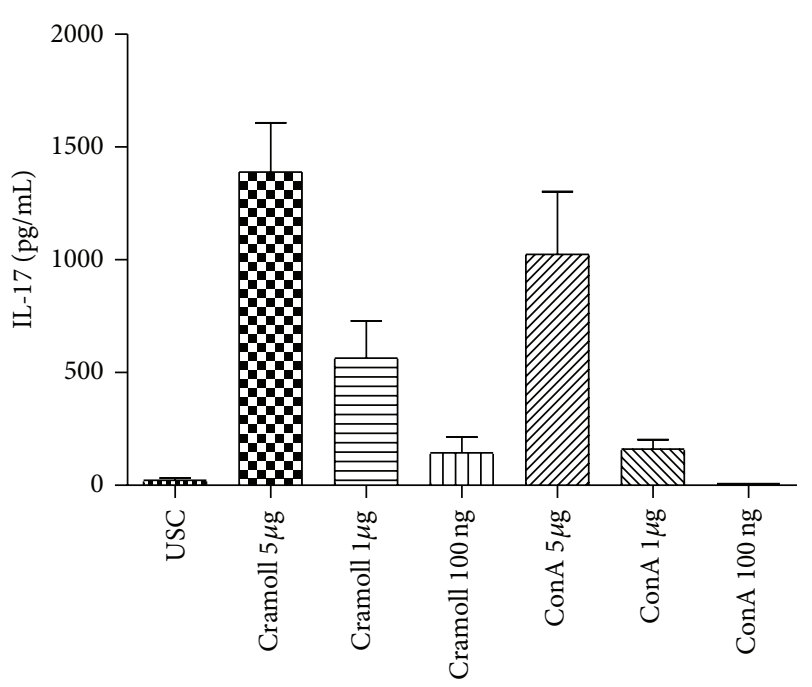

(b)

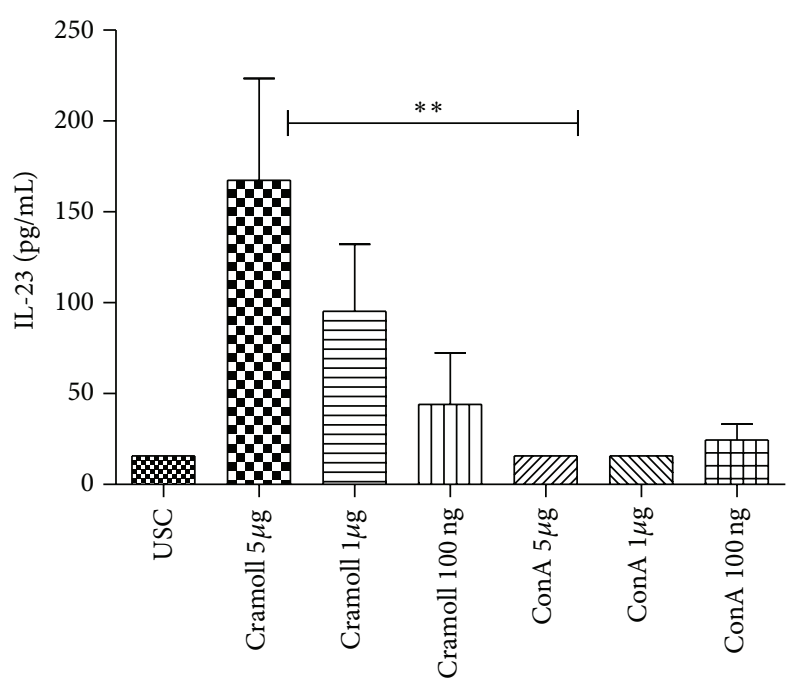

(d)

Figure 2: Production of IL-6 (a), IL-17 (b), IL-22 (c), and IL23 (d) in human PBMC culture stimulated with different concentration of Cramoll 1,4 and ConA.

showed also that in splenocyte cultures 3 different types of algae lectins Solieria filiformis (SfL), Pterocladiella capillacea (PCL), and Caulerpa cupressoides (CCL) induced high levels of IL-6. Stimulation with $1 \mu \mathrm{g} / \mathrm{mL}$ recombinant banana lectin (rBanLec) induced responses by $\mathrm{T}$ lymphocyte proliferation and intensive interferon-gamma, interleukin IL-4, and IL-10 secretion in splenocyte culture [24]. So far, this is the first paper in the literature using plant lectins as IL-17 and IL-22 inducers in splenocyte cultures.

Cramoll 1, 4 was effective in stimulating the production of IL- 6 better than ConA in human PBMC culture, however no significant difference was observed for IL-17. Concerning IL-22 stimulation Cramoll 1, 4 was more efficient than ConA and in relation to IL-23 showed a high significant difference $(P<0.01)$. This is the first paper to analyze Th17 cytokine path induction in human PBMCs where Cramoll 1, 4 already showed significant mitogenic activity compared with ConA and Phytohemagglutinin (PHA) [2]. Commonly lectins, mainly PHA and ConA, are used in research laboratories as inducing production of cytokines such as IL- $1 \beta$, TNF- $\alpha$, IL6 , IL-2, IFN- $\gamma$, and GM-CSF in culture of human PBMCs [25-27]. Here we present Cramoll 1, 4 as a biotechnological tool for Th17 related cytokine production and for the first time revealed this ConA property. The mechanism of the immunomodulatory action of Cramoll 1,4 is not totally known; Cramoll 1, 4 possibly induces the activation of $\mathrm{T}$ lymphocytes through transmembrane signals like ConA, since Cramoll 1, 4 has high homology with ConA [28, 29].

Our data showed that the second stimulus with ConA did not induce immunological memory in any assayed conditions for IL-6, IL-17A, IL-22, and IL-23 (Figure 3). Although no statistical difference has been observed between Cramoll 1 , 4 and ConA under the conditions studied, it is possible to verify that Cramoll 1, 4 induces a IL-6 dose-dependent 


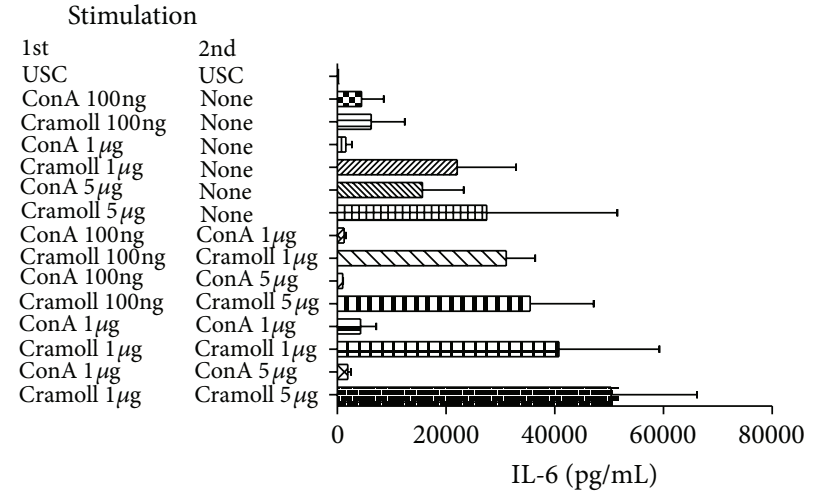

(a)

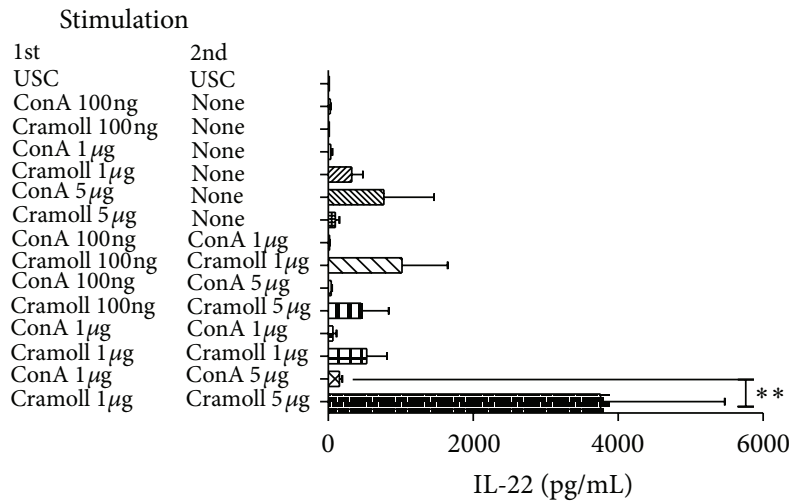

(c)

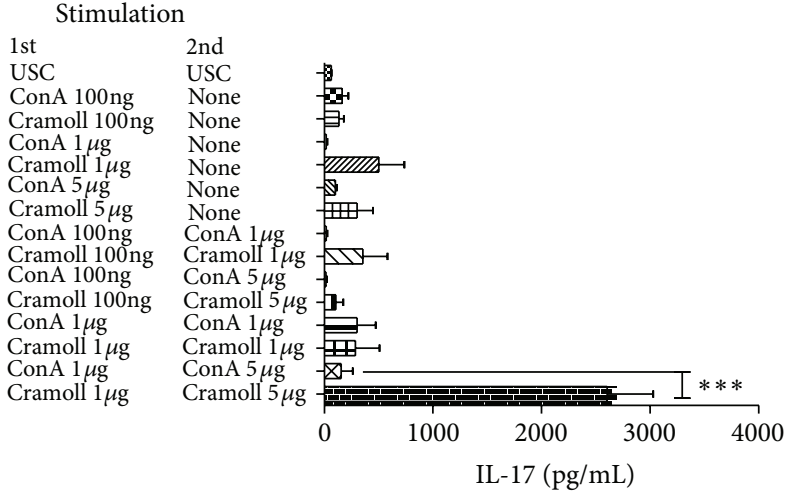

(b)

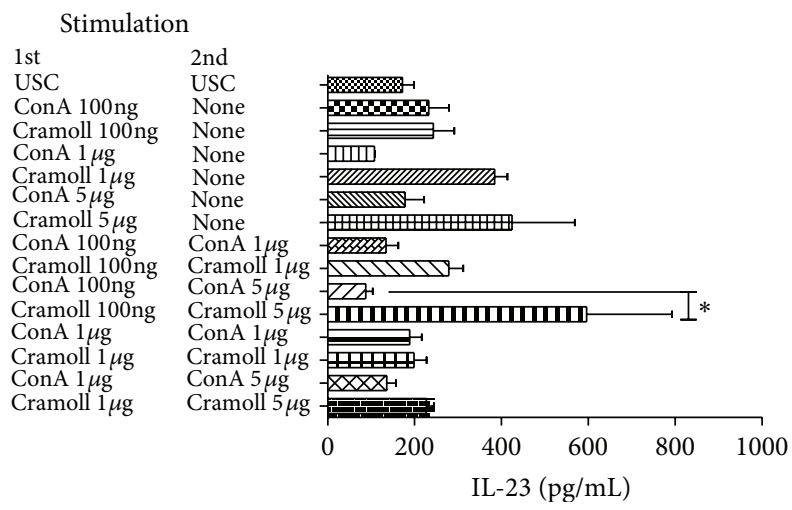

(d)

FIGURE 3: Analysis of immunological memory generation by different concentration of ConA and Cramoll 1, 4 in cultured human PBMC with the second stimulus being provided after five days of the first stimulus and cytokines measured after $24 \mathrm{~h}$.

response when used as single agent in all tested periods and when used at both time points (Figure 3(a)). These results are in agreement with previous studies from our group in which the first stimulus in vivo was carried out intraperitoneally (Cramoll 1,4 at $100 \mu \mathrm{L}$ ), and the second stimulation performed on splenocytes culture with Cramoll 1,4 at $10 \mu \mathrm{g} / \mathrm{mL}$. Then IL- 6 was measured $72 \mathrm{~h}$ later and six days later [14]. As shown in our results Cramoll 1, 4 was more efficient than ConA in the induction of the response via the IL-6; in both groups this cytokine production was significantly more efficient than that in the unstimulated group.

Cramoll 1, 4 induced significant immunological memory mediating the release of IL-17A and IL-22 when the first stimulus was performed at $5 \mu \mathrm{g} / \mathrm{mL}$ and the second at $1 \mu \mathrm{g} / \mathrm{mL}$; however only with IL-22 it was possible to observe a dose response. IL-23 results were slightly different than IL-17A and IL-22; the best dose for the first stimuli was $100 \mathrm{ng} / \mathrm{mL}$ and for the second was $5 \mu \mathrm{g} / \mathrm{mL}$. This may be related to the function of maintaining the phenotype Th17 by IL-23 [30] not requiring a large stimulus for pathway maintenance. So we can conclude that Cramoll 1, 4 is able to induce IL-6, IL$17 \mathrm{~A}, \mathrm{IL}-22$, and IL-23 cytokine production in vitro better than ConA, the most widely lectin used nowadays. Furthermore, the results suggested that Cramoll 1, 4 allowed immunologic memory generation by reinducing lymphocyte stimulation.

\section{Conflict of Interests}

The authors declare that they have no conflict of interests.

\section{Authors' Contribution}

P. Oliveira performed the human and BALB's culture, ELISA related experiments, and their data acquisition. M. Rêgo drafted the paper and performed the statistical analysis. R. Ramos and M. B. Cavalcanti gave support to P. Oliveira in experiments. M. T. Correia performed isolation and purification of Cramoll 1, 4 lectin. L. Coelho made isolation and purification of Cramoll 1, 4 lectin and revision of the paper. S. Galdino and M. Pitta elaborated, designed, and coordinated of the study, collaborated to the draft version of the paper, and gave final approval of submitted version of the paper. All authors read and approved the final paper. P. S. S. de Oliveira, M. J. B. de Melo Rêgo contributed in the same way.

\section{Acknowledgments}

This work was supported by the Fundação de Amparo a Ciência and Tecnologia do Estado de Pernambuco (FACEPE) and The Instituto Nacional de Ciência and Tecnologia para Inovação Farmacêutica (INCT_if). 


\section{References}

[1] N. Varejão, M. D. S. Almeida, N. N. T. de Cicco et al., "Heterologous expression and purification of a biologically active legume lectin from Cratylia mollis seeds (CRAMOLL 1)," Biochimica et Biophysica Acta, vol. 1804, no. 9, pp. 1917-1924, 2010.

[2] E. V. M. Maciel, V. S. Araújo-Filho, M. Nakazawa, Y. M. Gomes, L. C. B. B. Coelho, and M. T. S. Correia, "Mitogenic activity of Cratylia mollis lectin on human lymphocytes," Biologicals, vol. 32, no. 1, pp. 57-60, 2004.

[3] E. I. C. Beltrão, M. T. S. Correia, J. Figueredo-Silva, and L. C. B. B. Coelho, "Binding evaluation of isoform 1 from Cratylia mollis lectin to human mammary tissues," Applied Biochemistry and Biotechnology A, vol. 74, no. 3, pp. 125-134, 1998.

[4] A. L. de Lima, C. C. B. Cavalcanti, M. C. C. Silva et al., "Histochemical evaluation of human prostatic tissues with Cratylia mollis seed lectin," Journal of Biomedicine and Biotechnology, vol. 2010, Article ID 179817, 6 pages, 2010.

[5] C. M. de Melo, M. C. de Castro, A. P. de Oliveira et al., "Immunomodulatory response of Cramoll 1,4 lectin on experimental lymphocytes," Phytotherapy Research, vol. 24, no. 11, pp. 1631-1636, 2010.

[6] C. M. Melo, A. L. de Lima, E. I. C. Beltrão et al., "Potential effects of Cramoll 1,4 lectin on murine Schistosomiasis mansoni," Acta Tropica, vol. 118, no. 2, pp. 152-158, 2011.

[7] C. M. de Melo, C. S. Porto, M. R. Melo-Júnior et al., "Healing activity induced by Cramoll 1,4 lectin in healthy and immunocompromised mice," International Journal of Pharmaceutics, vol. 408, no. 1-2, pp. 113-119, 2011.

[8] C. A. S. Andrade, M. T. S. Correia, L. C. B. B. Coelho, S. C. Nascimento, and N. S. Santos-Magalhães, "Antitumor activity of Cratylia mollis lectin encapsulated into liposomes," International Journal of Pharmaceutics, vol. 278, no. 2, pp. 435-445, 2004.

[9] M. P. Fernandes, N. M. Inada, M. R. Chiaratti et al., "Mechanism of trypanosoma cruzi death induced by Cratylia mollis seed lectin," Journal of Bioenergetics and Biomembranes, vol. 42, no. 1, pp. 69-78, 2010.

[10] S. Vautier, M. D. G. Sousa, and G. D. Brown, "C-type lectins, fungi and Th17 responses," Cytokine and Growth Factor Reviews, vol. 21, no. 6, pp. 405-412, 2010.

[11] Z. Chen, A. Laurence, and J. J. O'Shea, "Signal transduction pathways and transcriptional regulation in the control of Th17 differentiation," Seminars in Immunology, vol. 19, no. 6, pp. 400408, 2007.

[12] S. Q. Crome, A. Y. Wang, and M. K. Levings, "Translational mini-review series on Th17 cells: function and regulation of human $\mathrm{T}$ helper 17 cells in health and disease," Clinical and Experimental Immunology, vol. 159, no. 2, pp. 109-119, 2010.

[13] M. T. S. Correia and L. C. B. B. Coelho, "Purification of a glucose/mannose specific lectin, isoform 1, from seeds of Cratylia mollis mart. (Camaratu Bean)," Applied Biochemistry and Biotechnology, vol. 55, no. 3, pp. 261-273, 1995.

[14] C. M. de Melo, H. Melo, M. T. S. Correia, L. C. B. B. Coelho, M. B. da Silva, and V. R. Pereira, "Mitogenic response and cytokine production induced by cramoll 1,4 lectin in splenocytes of inoculated mice," Scandinavian Journal of Immunology, vol. 73, no. 2, pp. 112-121, 2011.

[15] S. K. Bhutia, S. K. Mallick, and T. K. Maiti, "In vitro immunostimulatory properties of Abrus lectins derived peptides in tumor bearing mice," Phytomedicine, vol. 16, no. 8, pp. 776-782, 2009.
[16] S. Tripathi and T. K. Maiti, "Efficiency of heat denatured lectins from Abrus precatorius as immunoadjuvants," Food and Agricultural Immunology, vol. 15, no. 3-4, pp. 279-287, 2003.

[17] I. Crane, H. Leung, and S. Barwick, "The preparation of interferon gamma-producing T-cell hybridomas from jacalinstimulated T lymphocytes and the SH9 T-cell line," Immunology, vol. 53, no. 4, pp. 855-859, 1984.

[18] N. Pineau, P. Aucouturier, J. C. Brugier, and J. L. Preud'Homme, "Jacalin: a lectin mitogenic for human CD4 T lymphocytes," Clinical and Experimental Immunology, vol. 80, no. 3, pp. 420425, 1990.

[19] H. D. Dong, Y. Kimoto, S. I. Takai, and T. Taguchi, "Apoptosis as a mechanism of lectin-dependent monocyte-mediated cytotoxicity," Immunological Investigations, vol. 25, no. 1-2, pp. 65-78, 1996.

[20] C. Dong, “TH17 cells in development: an updated view of their molecular identity and genetic programming," Nature Reviews Immunology, vol. 8, no. 5, pp. 337-348, 2008.

[21] M. Mihara, M. Hashizume, H. Yoshida, M. Suzuki, and M. Shiina, "IL-6/IL-6 receptor system and its role in physiological and pathological conditions," Clinical Science, vol. 122, no. 4, pp. 143-159, 2012.

[22] C. M. de Melo, B. A. Paim, K. G. Zecchin et al., "Cramoll 1,4 lectin increases ROS production, calcium levels, and cytokine expression in treated spleen cells of rats," Molecular and Cellular Biochemistry, vol. 342, no. 1-2, pp. 163-169, 2010.

[23] T. M. Abreu, L. M. Silva, E. S. Vanderlei, C. M. de Melo, V. R. Pereira, and N. M. Benevides, "Cytokine production induced by marine algae lectins in BALB/c mice splenocytes," Protein and Peptide Letters, vol. 19, no. 9, pp. 975-981, 2012.

[24] M. M. Stojanović, I. P. Zivković, V. Z. Petrusić et al., "In vitro stimulation of $\mathrm{Balb} / \mathrm{c}$ and $\mathrm{C} 57 \mathrm{BL} / 6$ splenocytes by a recombinantly produced banana lectin isoform results in both a proliferation of T cells and an increased secretion of interferongamma," International Immunopharmacology, vol. 10, no. 1, pp. 120-129, 2009.

[25] D. de Groote, P. F. Zangerle, Y. Gevaert et al., "Direct stimulation of cytokines (IL- $1 \beta$, TNF- $\alpha$, IL-6, IL-2, IFN- $\gamma$ and GM-CSF) in whole blood. I. Comparison with isolated PBMC stimulation," Cytokine, vol. 4, no. 3, pp. 239-248, 1992.

[26] M. J. Micallef, T. Ohtsuki, K. Kohno et al., "Interferon- $\gamma$ inducing factor enhances $\mathrm{T}$ helper 1 cytokine production by stimulated human T cells: synergism with interleukin-12 for interferon- $\gamma$ production," European Journal of Immunology, vol. 26, no. 7, pp. 1647-1651, 1996.

[27] S. A. Stanilova, Z. G. Dobreva, E. S. Slavov, and L. D. Miteva, "C3 binding glycoprotein from Cuscuta europea induced different cytokine profiles from human PBMC compared to other plant and bacterial immunomodulators," International Immunopharmacology, vol. 5, no. 4, pp. 723-734, 2005.

[28] J. W. Hadden, "Transmembrane signals in the activation of Tlymphocytes by lectin mitogens," Molecular Immunology, vol. 25, pp. 1105-1112, 1998.

[29] G. A. de Souza, P. S. Oliveira, S. Trapani et al., "Amino acid sequence and tertiary structure of Cratylia mollis seed lectin," Glycobiology, vol. 13, pp. 961-972, 2003.

[30] B. Stockinger and M. Veldhoen, "Differentiation and function of Th17 T cells," Current Opinion in Immunology, vol. 19, pp. 281286, 2007. 

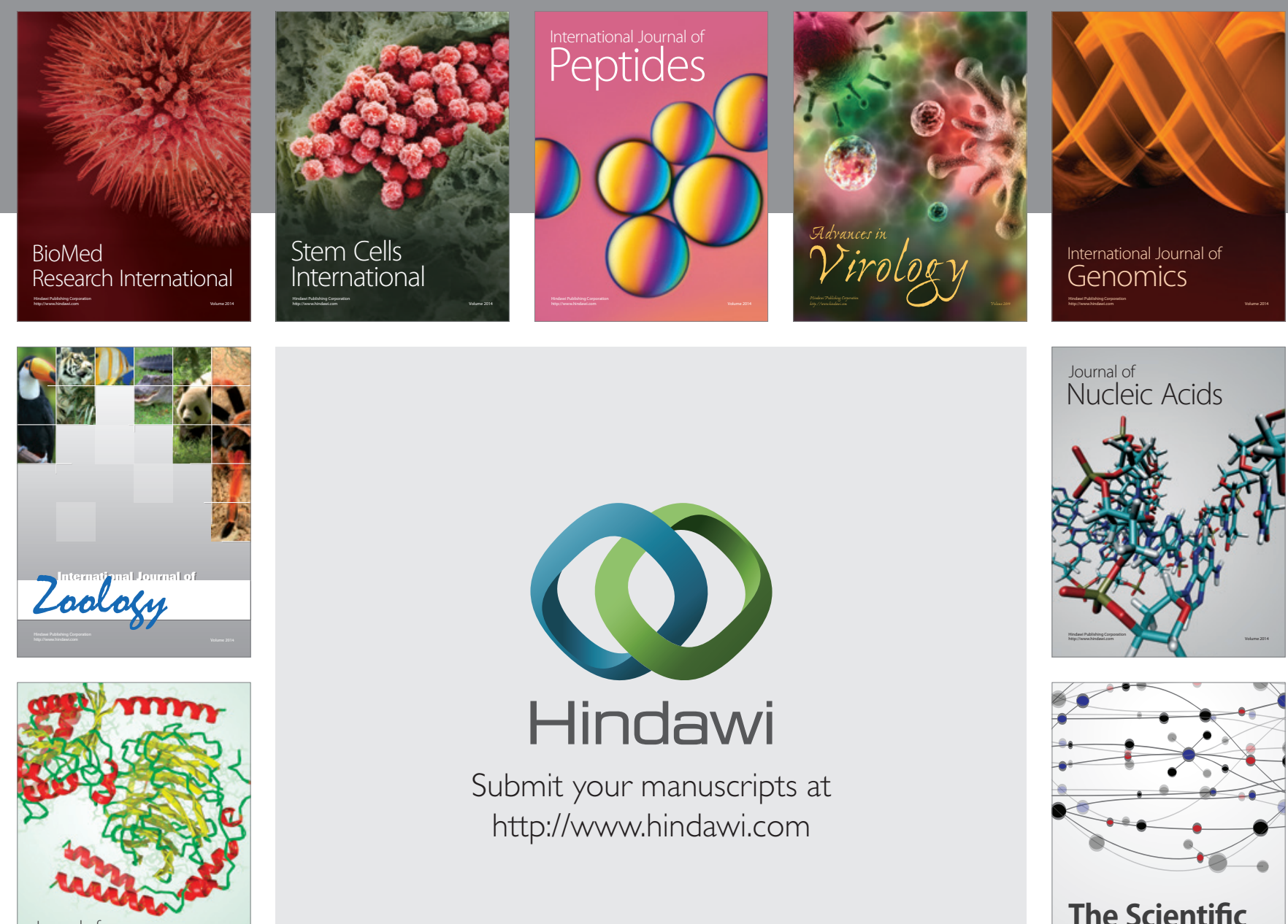

Submit your manuscripts at

http://www.hindawi.com

Journal of
Signal Transduction
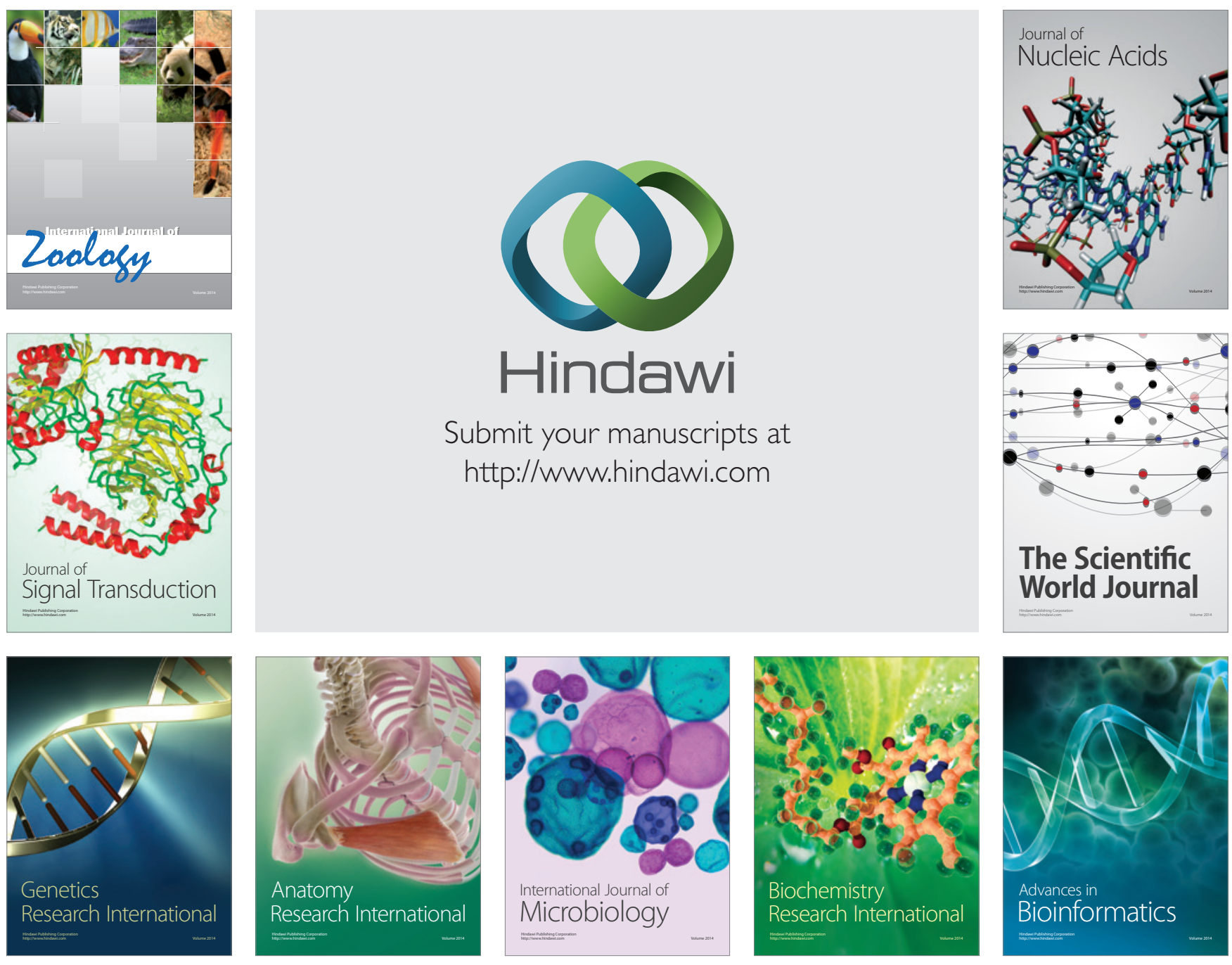

The Scientific World Journal
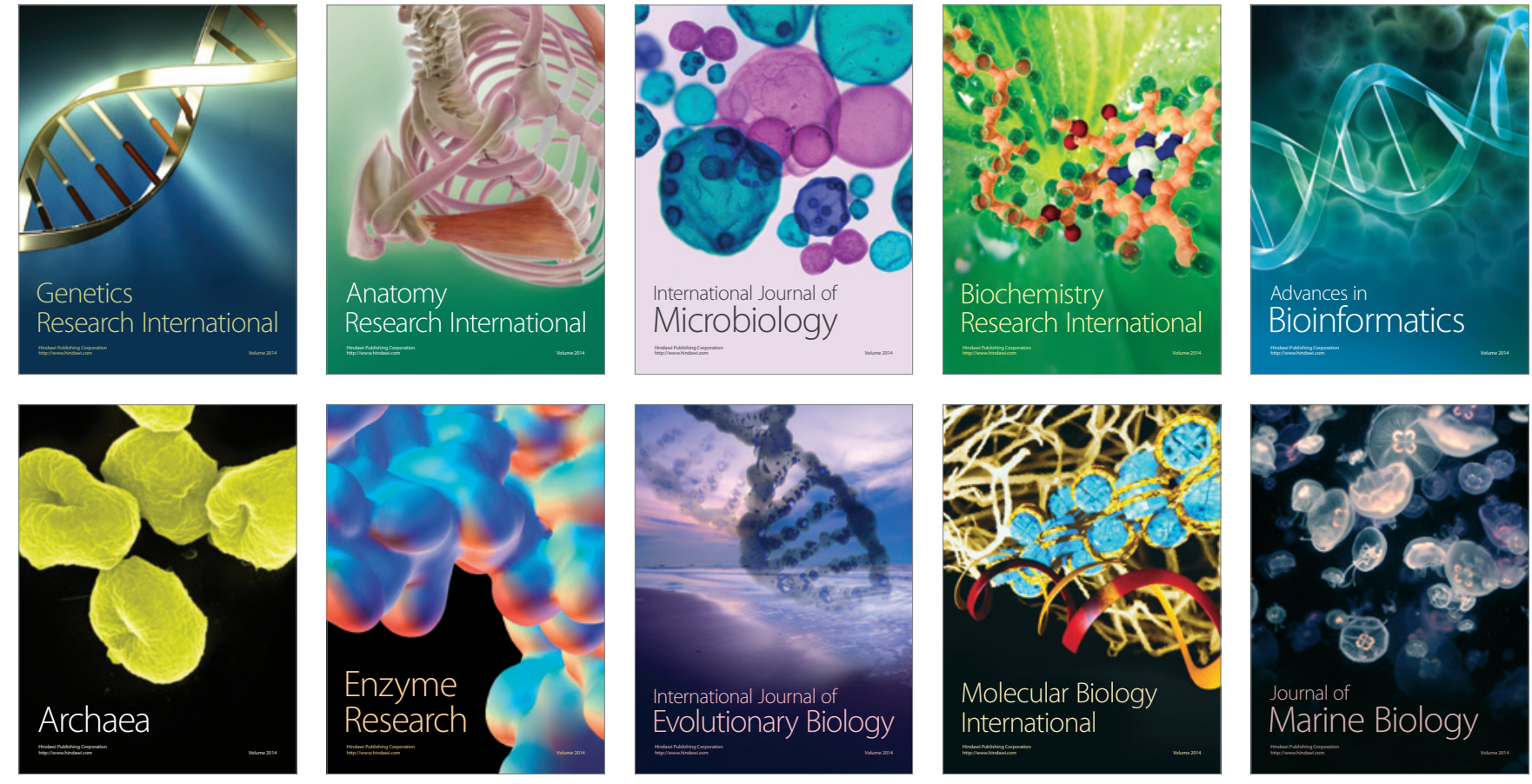